\title{
Comparing the effectiveness of acupressure and fish oil capsules and Ibuprofen on pain severity of primary dysmenorrhea
}

\author{
F. Behmanesh ${ }^{1^{*}}$, M. Zafari $^{2}$, M. Zeinalzadeh ${ }^{3}$, A. Aghamohammadi ${ }^{2}$ and S. Akbarpoor ${ }^{4}$ \\ ${ }^{1 *}$ Department of Midwifery, Babol University of Medical Sciences, Babol, Iran \\ ${ }^{2}$ Department of Midwifery, Islamic Azad University of Sari branch, Sari, Iran \\ ${ }^{3}$ Fatemeh Zahra Infertility and Reproductive Health research center, Babol University of Medical Sciences, Babol, Iran. \\ ${ }^{4}$ Department of statistics, Babol University of Medical Sciences, Babol, Iran. \\ ${ }^{1 *}$ corresponding author: PhD Student of Reproductive Health Email: midwifery@mubabol.ac.ir
}

\begin{abstract}
Dysmenorrhea is defined as cyclic pain directly related to menstruation and it is one of the common causes of sick leave and decline in women's quality of life. This study was conducted with the purpose of evaluating the effectiveness of acupressure and fish oil capsules on pain severity of primary dysmenorrhea. This clinical trial study was carried out on students of Azad University of Sari branch in 2011. Students afflicted with primary dysmenorrhea were randomly divided into three groups. The first group (60 students) received $1000 \mathrm{mg}$ of fish oil capsule every day for the duration of two successive cycles. In the second group (control group) 76 students were given $400 \mathrm{mg}$ Ibuprofen pills, as soon as the pain started, for two months; and in the acupressure group (60 students), the Saninjao point was pressed, at the start of the pain with the thumb for 20 minutes. The data was analyzed with SPSS software and the ANOVA, Friedman, Wilcoxon, Kruskal-wallis and Man-Whitney tests. Results showed a significant difference with respect to pain severity before and after the first and the second months of the interventions. Moreover, all three groups needed the same quantity of extra painkillers during the treatment duration. Acupressure and fish oil capsules are suitable treatment on pain severity of primary dysmenorrhea.
\end{abstract}

Keywords: Primary dysmenorrhea, Fish oil capsule, Acupressure, Pain relief.

Introduction

Primary dysmenorrhea defines as painful menstrual period in the absence of any underlying pathology (Smith et al., 2011). Dysmenorrhea, or painful menstruation, is a common gynecological disorder experienced by at least $50 \%$ of women during their fertility period. $10 \%$ of these women have such severe pain that it prevents them from attending their school or from going to their workplace. Therefore, women afflicted with dysmenorrhea are more often absent from their workplace and do worse in their studies than women who do not suffer from this disorder. Dysmenorrhea has two forms: primary and secondary. From the accounts given by the patients, and in clinical studies and tests, no clear background reasons have been found for primary dysmenorrhea, but secondary dysmenorrhea is accompanied by a background disorder (Yaghmaee et al., 2004; Zafari et al., 2011).

Prevalence of primary dysmenorrhea varies from 50 to $90 \%$ in different communities. In Iran, its prevalence has been reported to be from 74 to $86.1 \%$. Although primary dysmenorrhea does not threaten lives and does not cause any disfigurement, it can influence the quality of life of women and, in severe cases, it can disable them and make them inefficient that they cannot attend school or go to work (Dolatian \& Jaafari, 2004). Several reasons have been given for primary dysmenorrhea, the most agreed upon of which is the increase in the production of prostaglandins, and the role of these compounds in causing pain (Yaghmaee et al., 2004). For the treatment of primary dysmenorrhea, various methods have been

Research article

CIndian Society for Education and Environment (iSee)

"Dysmenorrhea"

http://www.indjst.org suggested: Aspirin, Acetaminophen, Ibuprofen, Naproxen and Mefenamic acid, oral contraceptive pill and cervix dilation in advanced cases, the use of IUDs, doing exercises, keeping the abdomen and back warm and using hot heating pad, electrical stimulus of nerves in the waist, abdomen and backside, using nutritional complements like calcium, magnesium, Vit E, Vit B1 and Vit $C$ and avoiding salt and cigarettes (Merrison et al., 1999; Zhang \& Liwan, 1999; Bernard et al., 2000; Kastap, 2000; Regidor et al., 2001; Sidani \& Campbell, 2002; Moghadamnia et al., 2010). On the other hand, the use of $\mathrm{CAM}^{*}$ has recently increased from the recent trends in medical care including a range of treatments with acupuncture and acupressure to prayer meetings and the use of herbal medicines (Behmanesh et al., 2010). Medications such as fish oil capsules in the treatment of dysmenorrhea appear to be most effective in treating dysmenorrhea.

Consumption of fish oil may cause the production of prostacyclin and reduces the intensity of dysmenorrhea. As one of the results obtained in this study, it can be said that more series 3 prostaglandins (PGE2, PGI3, and TXA2) are produced during menstruation; and that the use of fish oil causes the production of prostaglandins belonging to the prostacyclin class in the uterus, which brings about a reduction in the contraction of myometrium and in the contraction of the vessels of the uterus, which, in turn, decreases ischemia, and hence reduces pain (Zamani et al., 2005). 
Diagram 1. Participants through each stage of randomized, controlled trial.

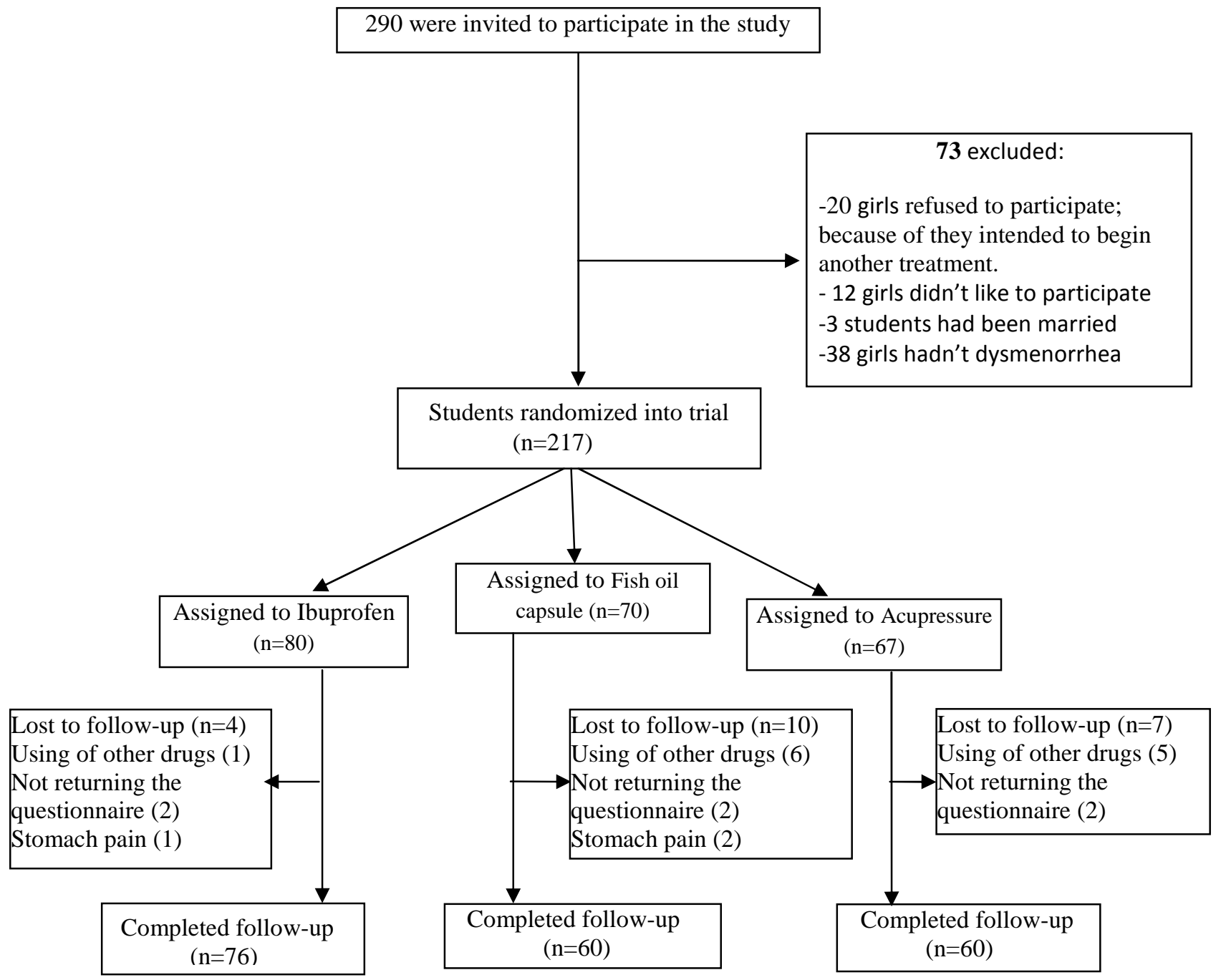

In acupressure, the technique of touching the body is used to balance the flow of energy in the body. The Saninjiao, the intersection of the three canals between the spleen, the kidneys, and the liver, is one of the most important points used in acupuncture. This point is situated 3 Kans above the inside ankle of the foot behind the hind edge of the tibia; and it is extensively used in curing gynecological, genitourinary, and digestive disorders, in the treatment of weakness and low blood pressure, in creating anesthesia during operations in the pelvic area, and in painless childbirth (Navidi et al., 2006). Various researches have shown that it can be an effective non-invasive nursing intervention for alleviation of primary dysmenorrhea (Jun et al., 2007).

Although the non-steroidal anti-inflammatory drugs are the drugs of choice for curing primary dysmenorrhea, and despite the fact that they are widely available without prescription, adolescents do not take advantage of effective treatment programs. Because of fears concerning the side effects of nonsteroidal antiinflammatory drugs and oral contraceptive pills, and based on a number of false beliefs, these types of drugs are not regularly used. Gastrointestinal effects (nausea, vomiting, and/or diarrhea) are of particular concern with NSAIDs. Effects are generally tolerable, but when treating women with risk factors for NSAID induced ulceration, the potential risks and benefits of using an NSAID should be considered (Proctor \& Farquhar, 2006). The purpose of this study is to compare the effectiveness of acupressure and fish oil capsules and Ibuprofen on pain severity of primary dysmenorrhea.

Materials and methods

This study was conducted as a randomized clinical trial. After getting written permission from the Ethics Committee of Babol University of Medical Sciences, this clinical trial was registered in the Iranian Registry of clinical Trials (www.IRCT.Ir [IR21101133004N4]).

The 176 students afflicted with moderate and severe primary dysmenorrhea ( $2 \& 3$ grads) (according to the Visual Analog Scale for Pain (VASP) of Andersch and Milsom (Andersch \& Milsom, 1982), were randomly allocated into three groups. The first group (60 students) 
received the daily dosage of $1 \mathrm{gm}$ of fish oil that produced by the American 21st Century company. The second group (76 students) was prescribed Ibuprofen produced by Alborz company, and for the third group (60 students) acupressure was used (Diagram1).

The inclusion criteria in this study were single, 18-22 years old student, with regular menstrual periods (every 26 to 30 days), had menstrual pains in most of the menstrual cycles in the past six months, and had mild to severe pain, according to the standard verbal multidimensional scale of Andersch and Milsom.

The exclusion criteria of the study included sensitivity to fish oil capsule, special diets (those undergoing hydrotherapy, vegetarians, uncooked-food eaters), doing any kind of regular exercise or attending special classes(sports classes, physical fitness classes, etc.), relaxation techniques during the past six months, any kind of diagnosed physical and mental illnesses or any type of genital diseases, history of surgery of the stomach or pelvic, addiction to cigarettes or alcohol, taking hormonal drugs or contraceptive pills, or having intense psychological or mental stresses in the duration of the study and if participants didn't do the interventions after starting them.

Every participant was studied for the duration of three menstrual cycles. For the first cycle (the control cycle) no treatment was given, and the participants were only asked to write down the features of the menstruation period with respect to the intensity of pain: they had to determine the intensity of their pain using the grading system of multi-dimensional speech.

The Visual Analog Scale for Pain is categorized in four grades. When the patients had 'no pain' it was scaled as ' 0 ', 'mild' and tolerable pain as ' 1 ', 'moderate and seriously disturbing' pain as ' 2 ' and 'severe and unbearable' pain as ' 3 ' (Rakhshaee, 2011). Then the participants were randomly assigned in three groups and treated, using the three methods, for the duration of two menstruation periods. The 60 students in the first group received $100 \mathrm{mg} /$ day of fish oil capsules for two months; the 76 students in the second group(control group) were prescribed $400 \mathrm{mg}$ lbuprofen pills, to be taken at the start

Table 1. Characteristic of the groups

\begin{tabular}{|c|c|c|c|}
\hline $\begin{array}{c}\text { Acupressure } \\
\text { Mean( SD ) }\end{array}$ & $\begin{array}{c}\text { Fish oil capsule } \\
\text { Mean(SD ) }\end{array}$ & $\begin{array}{c}\text { Ibuprofen } \\
\text { Mean(SD })\end{array}$ & P-value \\
\hline $21 / 17(1 / 18)$ & $20 / 13(1 / 44)$ & $20 / 13(1 / 51)$ & $0 / 11$ \\
\hline $12 / 13(1 / 15)$ & $11 / 97(1 / 27)$ & $12 / 69(1 / 03)$ & $0 / 92$ \\
\hline $14 / 8(1 / 07)$ & $14 / 2(1 / 78)$ & $14 / 77(1 / 20)$ & $0 / 14$ \\
\hline
\end{tabular}

of the pain, and be repeated $8 \mathrm{~h}$ later if the pain persisted and the 60 students in the third group were trained to press the Saninjiao point, at the start of the pain, with their thumb for 5 minutes (press the point for 6 seconds, release the pressure for 2 seconds, press for another 6 seconds, release the pressure for two seconds, and so on). After the 5 minutes, they had to change feet and repeat this process on the same point for 5 minutes. Altogether, this point was pressed for 20 minutes ( 2 times for 5 minutes on each foot). It must be added that each student was interviewed once a week to check upon the correct use of the medicine and the proper way of performing the acupressure. The fish oil capsule and Ibuprofen tablet were monthly given to the students, the possibility of the occurrence of side effects was explained to them, and they were asked to report any side effects they observed. At the end of two months, the groups receiving intervention were investigated with respect to the intensity of pain and extra pain killer.

Data analysis

The collected data were analyzed with SPSS software and ANOVA, Friedman, Wilcoxon, KruskalWallis and Man-Whitney tests. The ANOVA test used for comparing the demographic data such as age, menarche age and the age at the onset of dysmenorrhea. Because of knowing that the trend of pain intensity is different between the groups before and 1 month and 2 months after intervention or not, Friedman test was used and Wilcoxon test was used to distinguish differences between times. Kruskal-Wallis test was done to compare pain intensity between the three groups before and 1 month and 2 months after intervention. Then, the post hoc test of Man-Whitney, conducted for comparing two by two with correction Bonferroni (0/017). The use of extra painkiller by the participants and satisfaction from the used methods were evaluated through descriptive analysis.

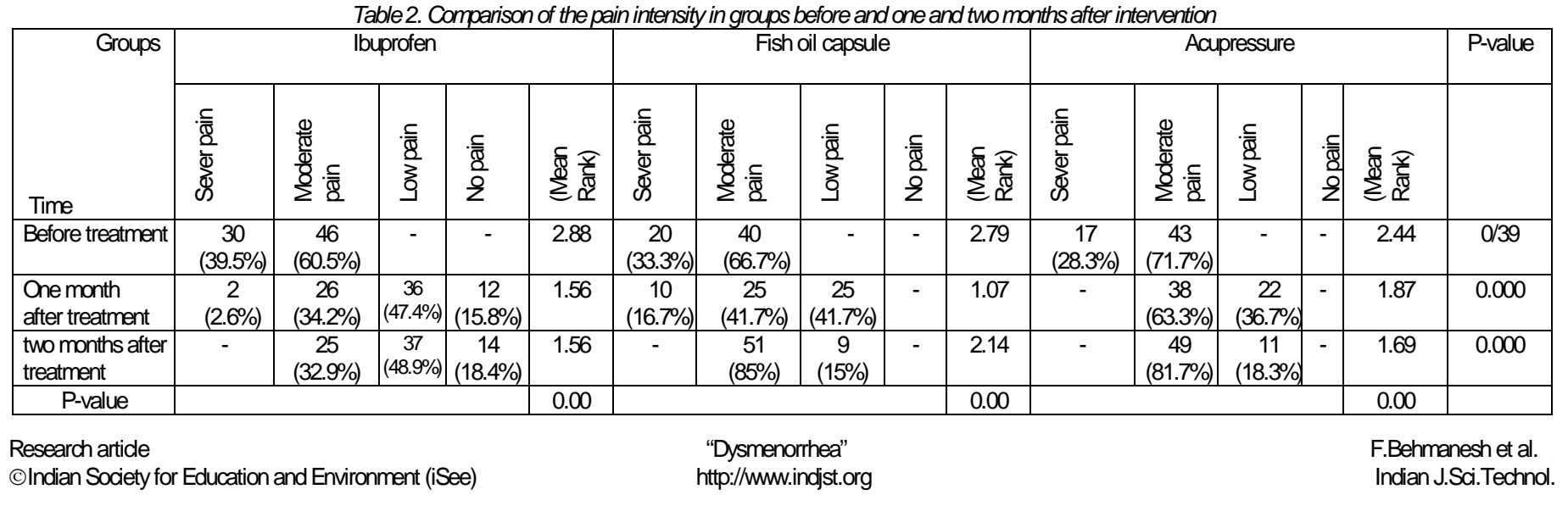




\section{Results}

There were no statistically significant differences among the three groups with respect to age, the Menarche age, participant age at beginning of dysmenorrhea and the pain intensity before treatment (Table1).

Friedman test showed a significant difference before intervention, 1month and 2 months after intervention in three groups. In all groups, Wilcoxon test showed that the dysmenorrhea pain reduced first month $(p<0 / 001)$ and second months $(p<0 / 01)$ after intervention compared to base severity pain of dysmenorrhea.

The Kruskal-Wallis test showed difference between three groups at first $(p<0 / 001)$ and second $(p<0 / 05)$ months after intervention (Table 2). But the MannWhitney post hoc test showed no differences between Ibuprofen and two other groups and the acupressure and fish oil capsule groups did not had any difference (Table 2). This finding showed that the pain relief effect of Ibuprofen was greater than fish oil and acupressure but as mentioned, these two methods too, reduced the pain intensity of dysmenorrhea.

Another finding of this study was that $13(17 / 1 \%)$ participants in Ibuprofen group, $14(23 / 3 \%)$ participants in fish oil capsule group and $17(28 / 3 \%)$ participants in acupressure group needed extra painkiller (all cases just used Ibuprofen as painkiller) and $x 2$ test did not show any differences between the groups. But there was a statistical significant difference in the degree of satisfaction from the used method in the three groups: in the Ibuprofen group, all of the participants, 76 (100\%) were satisfied from this method, in the acupressure group, 51 (85\%) girls were satisfied, $7(11 / 6 \%)$ were unsatisfied and $2(3 / 3 \%)$ did not have any idea and in the fish oil group, $30(50 \%)$ were satisfied, $12(20 \%)$ were unsatisfied and $18(30 \%)$ did not have any idea from this pain relief method. So the group taking Ibuprofen was more satisfied than the acupressure and the acupressure group was more satisfied than the fish oil group.

\section{Discussion}

In this study, the pain intensity decreased in the fish oil capsule group after treatment. Therefore, it can be concluded that fish oil has a considerable effect in reducing the intensity of pain in primary dysmenorrhea. In this respect, the results of this study are similar to those obtained by other studies (Dolatian \& Jaafari, 2004; Yaghmaee et al., 2004; Zamani et al., 2005; Wilson \& Murphy, 2001; Harel et al., 2011). In the study conducted by Wilson, fish oil (at 4 grams per day) was more effective than the placebo. We obtained these results with $1 \mathrm{~g} / \mathrm{day}$. Increasing the side effects such as: Vomiting and diarrhea; reduce the absorption of vitamin A. D.K.E, acceleration of glucose in diabetic person and Hemorrhagic infarcts, can accrue in high dose intake (more than $3 \mathrm{~g} /$ day). Fish oil is effective for healthy brain and painful menses (Zafari et al., 2011).

\section{Vol. 5 No. 6 (June 2012)}

ISSN: 0974- 6846

In another study, the effectiveness of treatment with fish oil and seal oil was compared with that of a combination of fish oil and vitamin B12, and it was found that the curative effect of fish oil and vitamin B12 was more suitable (Deutch et al., 2000).

Due to the relatively severe digestive side effects of nonsteroidal anti-inflammatory drugs (th in the 80 to $90 \%$ of the cases of dysmenorrhea), these drugs, despite their considerable curative effects, enjoy a low level of acceptability; and although they have been the most widely used treatment for dysmenorrhea so far, numerous studies have been carried out to find drugs with similar effects, but with fewer side effects (Poor esmail \& Ibrahim zadeh, 2002). So, we test acupressure for treatment of dysmenorrhea and it decreased the pain intensity. In this respect, these results were consistent with those obtained by Habek and Mehmet (2007), Sohrabi et al. (2006), and Kidney et al. (2001). After the intervention, the intensity of pain in the acupressure group was significantly reduced.

Pooresmael (2002), who carried out a study in this area, concluded that the three methods of acupressure, the use of placebo, and taking lbuprofen were effective in reducing primary dysmenorrhea, but that acupressure and Ibuprofen had very similar effects, which were considerably greater than those of the placebo.

Mahory et al. (1993) also conducted a study in the same area and stated that in the two groups of acupressure and Ibuprofen there was a significant difference in the intensity of pain before and after the treatments. Of course these differences did not considerably vary in the three consecutive months, but were very noticeable and important compared to the pain before the treatments. They also found that both the acupressure method and the use of painkillers were very effective in reducing pain, and reduced it equally (1993).

Kashefi et al. (2010) concluded that acupressure at Sanyinjiao point can be an effective, feasible, costeffective intervention for reducing pain of primary dysmenorrhea (2010). The effectiveness of acupressure, in Chinese traditional medicine is attributed to reinforcement of blood circulation and vital energy, which relieves cramping pain in the uterus. Melzack suggested that acupuncture's mechanism of pain relief is in that stimulation of large, myelinated fibers blocks the smaller fibers from transmitting painful stimuli (Chen \& Chen, 2004).

In another study carried out on 216 students of 14 to 18 years of age, in $50 \%$ of those in the acupressure group, and in $18 \%$ of those in the placebo group, the intensity of pain after the treatments was reduced to zero (Lee et al., 1999).

In the present study, $85 \%$ of participants in acupressure group were satisfied from this method for relieving their dysmenorrhea; whereas, all consumers of Ibuprofen were satisfied and fewer of them needed extra painkiller that its result was different from the others. But
Research article

CCIndian Society for Education and Environment (iSee)
"Dysmenorrhea"

http://www.indjst.org
F.Behmanesh et al. Indian J.Sci.Technol. 
in other studies, we saw the relieving effect of both acupressure and fish oil capsule in primary dysmenorrhea.

\section{Study limitations}

We could not do acupressure as an expert, so this method was done by the students themselves so the severity of pressure was different in participants. Although in some other studies, we saw that they taught acupressure to the students and asked them to do it (Bazarganipour et al., 2010. Wong \& Tse, 2010) We tell the students to take painkillers if they are in need.

\section{Conclusions}

Acupressure and fish oil capsules can be substitute for the non-steroidal anti-inflammatory drugs (which have many side effects) and OCPs in curing those afflicted with dysmenorrhea. It is also recommended that different dosages of fish oil capsule with different duration be used in further studies (Zafari et al., 2011).

\section{Acknowledgement}

The authors would like to thank the Research Deputy of Babol University of Medical Sciences for financially supporting the project, and to all who sincerely cooperated in the study.

\section{References}

1. Andersch B and Milsom I (1982) Epidemiologic study of young women with dysmenorrhea. Am. J. Obstet. Gynecol. pp: 144:655.

2. Bazarganipour $F$, Lamyian M, Heshmat R, Abadi MAJ and Taghavi A (2010) A randomized clinical trial of the efficacy of applying a simple acupressure protocol to the Taichong point in relieving dysmenorrhea. Int. J. Gynecol. \& Obstetrics. 111(2), 105-109.

3. Behmanesh F, Pasha H, Sefidgar AA, Moghadamnia AA and Ebrahimi Turi A (2010) Lavender and clotrimazol effect on the growth standard strains of $C$. albicans in vitro condition. JBUMS. 12(3), 26-31.

4. Bernard ND, Scilli AR, Hurlock D and Bertron P(2000) Diet and sex- Hormon binding globulin, Dysmenorrhea and premenstrual symptoms. Obstet. Gynecol. 95, 245-250.

5. Chen HM2 and Chen CH (2004) Effects of acupressure at the Sanyinjiao point on primary dysmenorrhea. J. Adv. Nursing. 48(4), 380-387.

6. Deutch B, Jorgensen EB and Hansen JC (2000) Menstrual disorder in Danish women reduced by dietary supplements of Omega - 3 PUFA and B12 (fish oil or seal oil capsules). Nutr. Res. 20, 621-631.

7. Dolatian M and Jaafari $\mathrm{H}$ (2004) Study of the effects of fish oil capsules on primary dysmenorrhea. J. Med. Univ Zanjan. 47, 7-13.

8. Habek D and Mehmet H (2007) Acupuncture treatment for prevention of early dysmenorrhea. Anesth Analg. 95(3), 632-635.

9. Harel Z, Biro FM, Kottenhan RK and Rosenthal SL (1996) Supplementation with omega-3 polyunsaturated fatty acids in the management of
Vol. 5 No. 6 (June 2012)

ISSN: 0974- 6846

dysmenorrhea in adolescents. Am. J. Obstet. Gyneacol. 174(4), 1335-1338.

10.Jun E, Chang S, Kang D and Kim S (2007) Effects of acupressure on dysmenorrhea and skin temperature changes in college students: A non-randomized controlled trial. Intl. J. Nursing Studies. 44(6), 973-981.

11. Kashefi F, Ziyadlou S, Khajehei M, Ashraf AR, Fadaee AR and Jafari $P(2010)$ Effect of acupressure at the Sanyinjiao point on primary dysmenorrhea: a randomized controlled trial. Complement Ther. Clin. Pract. 16(4), 198-202.

12. Kastap KE (2000) Nutrients and nutrition agent drug factors and comparisons. Transluted by Zargari A. 4th Edi. Tehran Publ. pp: 138-139.

13. Kidney M, Slade P and Randals CM (2001) Social support in women reporting dysmenorrhea. J. Psycosom. Re. 24, 79-84.

14. Lee A, Boehler M and Taerum T (1999) The use of non-pharmacological techniques to treatment of dysmenorrhea. Am. J. Pharmacol. 88, 1326-1329.

15. Mahory $p$ (1993) Acupressure \& its use for dysmenorrhea, Texas: Women Univ. Publ.

16. Merrison BW, Daniels SE, Kotey $\mathrm{P}$, Cantu $\mathrm{N}$ and Seidebery B (1999) RafeCoxibe aspecific Cyclooxygens-2 inhibitor in primary Dysmenorhea a randomized controlled trial. Obstetrics \& Gynecol. 94, 504-508.

17. Moghadamnia A, Mirhosseini N, Abadi MH, Omranirad A and Omidvar S (2010) Effect of Clupeonella grimmi (anchovy/kilka) fish oil on dysmenorrhea. East Mediterr. Health J. 16(4), 408-413.

18. Navidi AA, Khedmat $H$, Helli Saz MT, Naseri MH and Ghasemi Salmanie $\mathrm{KH}(2006)$ Basic principles and clinical acupuncture. Tehran: Tabib Publ.

19.Poor Esmail and Ibrahim Zadeh R (2002) Effect of acupressure and Ibuprofen on the severity primary Dysmenorrhea. J. Traditional Chinese Medic. 22(3), 205-210.

20.Proctor $M$ and Farquhar $C$ (2006) Diagnosis and management of dysmenorrhea. BMJ. 332(7550), 1134-1138.

21.Rakhshaee Z (2011) Effect of three yoga poses (cobra-cat and fish poses) in women with primary dysmenorrhea: A randomized control trial. J. Pediatric Adolescent Gynosol. 24, 192-196.

22. Regidor PA, Regidor M and Rows S (2001) Prospective randomized study comparing the GnRHagonist and lynesternol in treatment Sever endometrosis. Gynecol. Endocrynol. 15, 202-209.

23.Sidani $M$ and Campbell J (2002) Select topics. Primary care clinic in office practice: Gynecol. 27(2), 247-327.

24.Smith CA, Crowther C, Petrucco S, Justin Beilby J and Dent H (2011) Acupuncture to treat primary dysmenorrhea in women: A randomized controlled trial. Evidence-Based Complementary \& Alternative
Research article

(C)Indian Society for Education and Environment (iSee)
"Dysmenorrhea"

http://www.indjst.org
F.Behmanesh et al. Indian J.Sci.Technol. 
Medic. Article ID 612464, 11 pages. doi:10.1093/ecam/nep239.

25.Sohrabi Z, Tadayon M and Javadifar N (2006) Comparison the effect of acupressure and Ibuprofen on dysmenorrhea Available from: URL: http://www.Sid.ir.

26. Wilson ML and Murphy PA (2001) Herbal and dietary therapies for primary and secondary dysmenorrhea. Cochrane Database Sys. Rev. 97(36), pp: 44.

27.Wong C, Lai K Y and Tse HM (2010) Effects of SP6 acupressure on pain and menstrual distress in young women with dysmenorrhea. Complementary Therapies in Clinical Practice. 16(2), 64-69.

28. Yaghmaee M, Moradi A and Hosseini R (2004) Comparison of the effects of mefenamic acid with mefenamic acid and fish oil in the reduction of pain in primary dysmenorrhea. J. Med. Univ. Gilan. 13(49), 68-72.

29.Zafari M, Tofighi M, Aghamohammady A, Behmanesh $F$ and Rakhshaee Z (2011) Comparison of the effect of acupressure, fish oil capsules and ibuprofen on treatment of primary dysmenorrhea. Afr. J. Pharmacy \& Pharmacol. 5(8), 1115-1119.

30.Zafari M, Behmanesh $\mathrm{F}$ and Agha Mohammadi A (2011) Comparison of the effect of fish oil and Ibuprofen on treatment of severe pain in primary dysmenorrhea. Caspian J. Internal Medic. 2(3), 279282.

31.Zamani M, Nasrollahi S and Kashani K (2005).The curative effects of fish oil on primary dysmenorrhea. $J$. Med. Univ. Gorgan. 17(1), 39-42.

32.Zhang WY and Liwan A (1999) Efficacy of minor analgesic primary Dysmenorrhea: a systematic review. Br. J. Obstet Gynaecol. 1998, 105, 280-289. 